\title{
Mass Indicator
}

National Cancer Institute

\section{Source}

National Cancer Institute. Mass Indicator. NCI Thesaurus. Code C154886.

An indication as to whether a mass is present. 